\title{
Conducta y actitud ambiental responsable en estudiantes universitarios en Lima, Perú
}

\section{Responsible environmental behavior and attitude in university students in Lima, Peru}

\author{
Edith Olivera Carhuaz $^{1 \mathrm{a}}$; Víctor Pulido Capurro ${ }^{2}$; Daniel Yupanqui-Lorenzo ${ }^{3}$ \\ Universidad Jaime Bausate y Meza, Lima, Perú ${ }^{1}$ \\ Universidad Privada San Juan Bautista-Escuela de Medicina Humana, Lima, Perú2 \\ Universidad Jaime Bausate y Meza, Lima, Perú ${ }^{3}$ \\ (iD) Orcid ID: https://orcid.org/0000-0002-7400-8625 \\ (iD) Orcid ID: https://orcid.org/0000-0002-9238-5387² \\ (iD) Orcid ID: https://orcid.org/0000-0002-8977-2888 3
}

Recibido: 20 de agosto de 2020

Aceptado: 29 de octubre de 2020

\section{Resumen}

La actitud hacia el ambiente constituye un indicador de la conducta ambientalista y se manifiesta en la acción y las regulaciones proambientales. El objetivo de este estudio fue conocer la relación entre la conducta y la actitud ambiental responsable en estudiantes universitarios en Lima, Perú. El enfoque fue cuantitativo, con diseño descriptivo-correlacional y la muestra estuvo conformada por 287 estudiantes hombres y mujeres de diversas carreras. Los instrumentos utilizados fueron dos escalas que miden la actitud ambiental: percepción de problemas ambientales, percepción de razones para problemas ambientales; intención de conductas proambientales y, tipos de preocupación ambiental; las mismas que evalúan de manera independiente la conducta proambiental. Se determinó la relación positiva entre la conducta y actitud ambiental responsable, así como entre los factores componentes de las variables.

Palabras claves: actitud ambiental; conducta ecológica; medio ambiente; ecología; universitarios.

\footnotetext{
${ }^{\text {a }}$ Correspondencia al autor

E-mail: esoc_olicar@hotmail.com
} 


\begin{abstract}
Attitude towards the environment is an indicator of environmental behavior and is manifested in the action and pro-environmental regulations. The purpose of this study was to know the relationship between behavior and responsible environmental attitude in university students in Lima, Peru. The approach was quantitative, with a descriptive-correlational design, and the sample consisted of 287 male and female students from different careers. The instruments used were two scales that measure the environmental attitude: perception of environmental problems, perception of reasons for environmental problems; intention of pro-environmental behaviors and, types of environmental concerns; the same ones that independently evaluate the attitude and proambiental behavior. The positive relationship between responsible environmental behavior and attitude was determined, as well as between the component factors of the variables.
\end{abstract}

Keywords: environmental attitude; ecological conduct; environment; ecology; university students.

\title{
Introducción
}

Desde las últimas décadas del siglo pasado, la conservación y el cuidado del ambiente constituye una prioridad fundamental, que se ve reflejada en las políticas de la mayor parte de los países. Se han desarrollado tecnologías limpias, implementado políticas y promulgado una legislación orientada a promover el desarrollo sostenible de los recursos naturales (Álvarez-Lires et al., 2017). Más aun, se han celebrado cumbres como la Conferencia de las Naciones Unidas, sobre el medio humano, Estocolmo, desarrollada en Estocolmo, 5 a 16 de junio de 1972, donde se concluyó que la educación debe contribuir a generar conciencia y coadyuvar a la toma de decisiones a favor del cuidado del ambiente; la Conferencia de Tbilisi en 1977, en la cual se precisó la función de la educación ambiental y su inmersión dentro de los estudios considerados formales, con el propósito de construir una estrategia que conlleve a lograr los objetivos establecidos en la Carta de Belgrado de 1975; y otros eventos de notable relevancia como el Congreso Internacional de Educación y Formación sobre Medio Ambiente en Moscú, 1987; la Cumbre de la Terra en Río de Janeiro, 1992; la Conferencia Internacional sobre Medio Ambiente y Sociedad: Educación y Sensibilización para la Sostenibilidad, Tesalónica, 1997; la Conferencia de Río en Sudáfrica, 1997, la Cumbre Mundial para el Desarrollo Sostenible, Johannesburgo, 2002. (UNESCO, 1978; Villadiego-Lordy et al. 2017).

Posteriormente, los países latinoamericanos impulsados por la Carta de Belgrado desarrollaron un conjunto de estrategias a fin de lograr los objetivos referidos, los cuales involucraban: generar conciencia, impulsar conocimientos, promover actitudes y aptitudes en la población. (UNESCO, 1975; Calixto, 2012). A pesar de los esfuerzos realizados, las actividades 
humanas que generan el 70\% de las emisiones de gases de efecto invernadero están destinados al uso energético: 24\% para la generación de energía, 14\% al sector industrial, $8 \%$ en el uso comercial y residencial, 14\% para el transporte y el 10\% restante en otros usos energéticos, con evidentes consecuencias en el calentamiento global del planeta (World Bank, IFC \& MIGA. 2016; Choon et al., 2019; Yilmaz \& 2020). La contaminación ha puesto en riesgo la salud de las personas y atenta contra la calidad de vida (Pulido, 2017). Cada día, se generan toneladas de residuos en todo el mundo, y son pocos los países que plantean propuestas objetivas de mejora y por ende contribuir a la concienciación de su población (Bermúdez et al., 2011; Leiva, 2020). Es más, la situación se ha agudizado en estos últimos años, con la pérdida de miles de hectáreas en la Amazonía debido a los incendios forestales, así como la deforestación para fines agrícolas y ganaderos, lo que viene causando un enorme impacto social con la consecuente desaparición de especies de animales y plantas (Dourojeanni, 2019).

En efecto, los problemas ambientales, no solo se ven influenciados por el accionar de las autoridades que trabajan en la protección del entorno, sino en el escaso conocimiento, el inadecuado comportamiento de los ciudadanos y las actitudes de indiferencia que agravan el problema (Rivera-Jacinto \& Rodríguez-Ulloa, 2009; Espino-Román et al., 2015) de manera que la participación de la ciudadanía en la conservación del ambiente es imprescindible (Pulido \& Olivera, 2018). En este escenario, no queda duda que la educación ambiental juega un rol fundamental frente a las amenazas ambientales que atentan contra la supervivencia del hombre en el planeta. De allí la importancia de que en la educación superior se implementen estrategias activas que permitan evidenciar cambios de actitud y comportamientos en los individuos (Olaguez-Torres et al., 2019).

La actitud, desde una concepción tridimensional, engloba tres aspectos fundamentales: las creencias, las emociones y las tendencias conductuales; las mismas que influyen en el comportamiento humano, además de formar parte de la intención, junto con las normas subjetivas y el control conductual percibido (Yupanqui, 2017); de modo que, en conjunto influencian en el proceder del sujeto (Albalá \& Maldonado, 2018). Por otra parte, la intención conductual es la probabilidad subjetiva de que una intención se convierta en acción (Barreto \& Neme, 2014)

En ese orden de ideas, es importante investigar la actitud hacia el ambiente tomando como un indicador la conducta ambientalista y, por otro lado, la intención comportamental por su valor predictivo (Amérigo et al., 2012; Amérigo et al., 2017), sin embargo, son pocos los estudios 
realizados a nivel universitario (Álvarez \& Vega, 2009). En congruencia con todo lo señalado, el presente estudio tuvo como objetivo conocer la conducta y las actitudes ambientales responsables en estudiantes de una universidad privada de Lima, Perú.

\section{Materiales y métodos}

El estudio es de tipo cuantitativo y nivel descriptivo correlacional, en el que se analizó la relación entre las variables estudiadas y su efecto (Hernández \& Mendoza, 2018). Se evaluó una muestra de estudiantes universitarios de las Escuelas de Humanidades, Sociales, Ingenierías y Ciencias de la Salud que pertenecen a una universidad privada de Lima Metropolitana. La muestra asciendió a 287 estudiantes, 80 hombres (27.9\%) y 207 mujeres (72.1\%). El muestreo empleado fue no probabilístico intencional, para el que se establecieron criterios de inclusión y exclusión.

Los instrumentos utilizados provienen de una escala sobre actitudes hacia el medio ambiente desarrollado por los autores de la investigación. Estos instrumentos son: Actitud ambiental responsable y Conductas proambientales. Se realizó para ambas escalas un análisis previo para obtener evidencia de validez por medio del constructo. Para la Actitud ambiental responsable (AAR), se realizó un análisis de ítem-test con la finalidad de estudiar las correlaciones. De este resultado se procedió a eliminar dos ítems (8 y 11) que no mostraban significancia en su relación con la prueba. El proceso de AFE (KMO: 0.924; p<.05) determinó que el instrumento mantiene estabilidad en los datos y se segmentaron en 5 factores. Por otro lado, el análisis de consistencia interna obtuvo un coeficiente de 0,88 alfa de Cronbach, lo cual favorece al uso del instrumento. Por otro lado, se realizó el mismo proceso para el instrumento de Conductas proambientales (CP). El AFE (KMO: 0.863; p<.05), se agrupó en 3 factores representativos. Además, la consistencia interna mediante el estadístico alfa de Cronbach resultó ser de 0,804. Ambos instrumentos mostraron fiabilidad, evidencia de validez por medio del constructo; y a través de su análisis factorial exploratorio.

\section{Resultados}

De acuerdo con los resultados obtenidos, se presentan dos tipos de análisis según el diseño propuesto. Primero se analiza descriptivamente las variables y luego la correlación entre variables y sus factores. En relación con la tabla 1, la actitud ambiental responsable presenta una media de 58.20, el cual se encuentra dentro de un nivel promedio, en tanto que los estudiantes poseen 
posturas tanto desfavorables como favorables respecto al medio ambiente. Sobre la conducta proambiental, se obtuvo una media de 42.81, que corresponde a un rango medio, lo que determinó que el estudiante mantuvo un análisis previo sobre la situación actual y la problemática ambiental, lo cual juega un rol importante en el sentido de que impulsa, aunque de forma leve en acciones que pueden favorecer el cuidado y la conservación del ambiente. Esa percepción de los estudiantes universitarios no concuerda en su totalidad con la visión actual de los problemas actuales ambientales, la necesidad del desarrollo sostenible y ecológicamente sustentable y la adopción de una actitud ambiental responsable ocupan el centro de las preocupaciones de la sociedad contemporánea (Torres-Maya et al., 2019). Además, se observa que el índice de K-S mantuvo un nivel de significancia por debajo de 0.05 , lo cual permite rechazar el supuesto de normalidad de los puntajes; en ese sentido, se consideró conveniente utilizar una prueba no paramétrica como el Rho de Spearman, para obtener los índices de correlación.

\section{Tabla 1}

Análisis descriptivo de la AAR y CP

\begin{tabular}{lcccc} 
Escala/Factores & M.E. & D.E. & Pc & K-S \\
& & & {$[25-75] \%$} & \\
\hline Actitud ambiental responsable (AAR) & 58.20 & 6.41 & $55-63$ & 0.00 \\
Importancia de la vegetación (AAR-D1) & 13.28 & 1.75 & $12-14$ & 0.00 \\
Problema de contaminación del aire (AAR-D2) & 17.61 & 2.48 & $16-20$ & 0.00 \\
Cuidado del medioambiente (AAR-D3) & 10.17 & 1.44 & $9-11$ & 0.00 \\
Dificultades en el cuidado ambiental (AAR-D4) & 7.15 & 1.03 & $7-8$ & 0.00 \\
Las personas como gestores del problema ambiental (AAR- & & & & \\
D5) & 7.12 & 1.14 & $6-8$ & 0.00 \\
Conductas proambientales (CP) & 42.81 & 5.13 & $39-47$ & 0.00 \\
Acciones comunales (CP-D1) & 10.08 & 1.51 & $9-11$ & 0.00 \\
Conservación ambiental (CP-D2) & 18.66 & 2.84 & $17-21$ & 0.00 \\
Conductas de prevención proambiental (CP-D3) & 14.07 & 1.60 & $13-15$ & 0.00
\end{tabular}

Las relaciones expuestas en la tabla 2, demuestran que en las variables estudiadas AAR y $\mathrm{CP}$, mantienen una relación estadísticamente significativa con un coeficiente de 0.563 ; lo que se 
caracteriza como una relación moderada con tendencia alta. Luego se expone, el análisis correlacional del AAR con los factores de CP; donde se determinó que todos los factores se relacionan a nivel moderado con la actitud hacia el medio ambiente, y que todas las relaciones se muestran con un nivel de significancia por debajo del 0.01. Por un lado, estos resultados refutan lo propuesto por Vicente-Molina et al. (2013) quienes argumentan que la actitud no es un variable que sirva como un antecedente de importancia en la conducta proambiental. Por otro lado, la motivación junto con la actitud puede ser determinantes para la intención conductual; la intensidad y la dirección de la motivación es un factor que beneficia la conducta (Vila-Tojo et al., 2019).

\section{Tabla 2}

Análisis correlacional de la actitud ambiental responsable y la conducta proambiental con factores

\begin{tabular}{llllll} 
Variables & AAR & CP & CP-D1 & CP-D2 & CP-D3 \\
\hline AAR & 1 & & & \\
CP & $.563 * *$ & 1 & & \\
CP-D1 & $.511 * *$ & $.801 * *$ & 1 & & \\
CP-D2 & $.469 * *$ & $.909 * *$ & $.584 * *$ & 1 & \\
CP-D3 & $.520 * *$ & $.830 * *$ & $.633 * *$ & $.603 * *$ & 1
\end{tabular}

**p<.01; AAR: Actitud ambiental responsable; CP: Conducta proambiental; CP-D1: Acciones comunales; CP-D2: Conservación ambiental; CP-D3: Conductas de prevención proambiental.

Respecto a la tabla 3, se muestra que la relación entre la CP y los factores de AAR, se relacionan con un nivel de significancia por debajo de 0.01; sin embargo, el factor de Importancia de la vegetación (AAR-D1) muestra correlación débil pero estadísticamente significativo, quedando demostrado el bajo nivel de importancia para relacionarse con la conducta ambiental responsable. Por otro lado, los demás factores se muestran con un nivel moderado de su coeficiente de relación. 


\section{Tabla 3}

Análisis correlacional de las conductas proambientales y los factores de AAR

\begin{tabular}{lllllll} 
Variables & CP & AAR-D1 & AAR-D2 & AAR-D3 & AAR-D4 & AAR-D5 \\
\hline CP & 1 & & & & \\
AAR-D1 & $.360^{* *}$ & 1 & & & \\
AAR-D2 & $.411^{* *}$ & $.442^{* *}$ & 1 & & & \\
AAR-D3 & $.533^{* *}$ & $.475^{* *}$ & $.477^{* *}$ & 1 & \\
AAR-D4 & $.478^{* *}$ & $.474^{* *}$ & $.563^{* *}$ & $.566^{* *}$ & 1 \\
AAR-D5 & $.419^{* *}$ & $.474^{* *}$ & $.490^{* *}$ & $.494 * *$ & $.609 * *$ & 1
\end{tabular}

** $\mathrm{p}<.01, * \mathrm{p}<.05 ; \mathrm{CP}$ : Conducta proambiental; AAR-D1: Importancia de la vegetación; AAR-D2: Problema de contaminación del aire; AAR-D3: Cuidado del medioambiente; AAR-D4: Dificultades en el cuidado ambiental; AAR-D5: Las personas como gestores del problema ambiental.

\section{Discusión}

El estudio determinó que existe una relación estadísticamente significativa y moderada $($ rho $=.563)$ entre las variables conducta y actitud ambiental responsable, por lo que los resultados obtenidos son similares a los encontrados por (Herrera-Mendoza et al., 2016; Campos et al. 2015; Cortés-Peña 2016; Palacios et al., 2015), sin embargo, algunas investigaciones demostraron resultados contrarios (Rivera-Jacinto \& Rodríguez-Ulloa, 2009). Desde esa perspectiva hay que señalar que la cultura tiene un rol importante en la formación de los elementos de la orientación de la conducta, así como de la actitud ambiental responsable con respecto al cuidado y la conservación del medio natural de acuerdo con la actividad que desarrollan los individuos en el entorno donde se desenvuelven (Torres, Suárez \& Dueñas, 2016).

El involucramiento del estudiante, en programas de educación con un fuerte contenido ecológico, tiene implicancias directas en la generación de conciencia ambiental, lo que le permite comprender y analizar, con mayor facilidad la situación actual de la conservación de los recursos naturales en el mundo. Una de las propuestas expresada por Gomera (2008) fue que en las universidades se deben impulsar propuestas para la ambientalización de la universidad: programas 
sociales, experiencias interuniversitarias, entre otros. Además, proponer situaciones reales y vivenciales para que el estudiante module su postura actitudinal hacia el cuidado del planeta.

El hecho de mantener conductas de prevención y conservación del ambiente, genera en el estudiante una estrecha relación con la actitud, puesto que la misma forma parte de una predisposición, basado en creencias y afectos de un hecho; si bien los resultados han demostrado la relación entre la conservación, prevención e involucramiento de terceros a un objetivo común, del mismo modo, se evidencia que la intención, mantiene influencia sobre la conducta (Lucie, 2010). Así mismo, la formación de la conciencia ecológica es fundamental para promover el establecimiento de una cultura de conservación ambiental y en el largo plazo, consolidar la visión del desarrollo sostenible, basado en valores que promuevan una conducta éticamente responsable. En ese sentido, el trabajo de Sevillano \& Olivos (2019) demuestra que el efecto de las normas descriptivas a favor del medio ambiente puede generar conductas en beneficio de su cuidado y mantenimiento apropiado. Por ello, la aplicación de normas en el entorno educativo superior resulta positivo para un cambio conductual (Onokala, Banwo \& Okeowo, 2018; Chakraborty et al., 2017); aún más, se destaca que la psicología ecológica resulta beneficiosa para el aprendizaje de nuevos modos de conductas a favor del ambiente.

Con respecto, a los factores referidos al cuidado del medioambiente, la dificultad en el cuidado ambiental y la actuación de las personas como gestores del problema ambiental, se encontraron índices correlacionales moderados con la conducta proambiental. Así mismo es preciso señalar que las actitudes de los estudiantes hacia el ambiente, parten de una visión constituida por elementos como el agua, el aire, las plantas, los animales y los árboles, que son esenciales para la vida del ser humano y por ello deben ser conservados; sin embargo, este cuidado trasciende la responsabilidad individual y valora la responsabilidad que se asume como sociedad (Calixto, 2012; Medina et al., 2017). Dicha actitud puede estar relacionada de tal manera que está intrínsecamente vinculada a formar parte de la estructura de la intención conductual (Olivera, 2020). Pero también, hay que tener en cuenta que la moral, la consciencia y la mentalidad, están constituidas por una serie de valores que influyen en el comportamiento proambiental (Janmaimool \& Khajohnmanee, 2018); lo que provocaría una mayor atención sobre el cuidado de los animales, el mantenimiento de la vegetación y la limpieza del ornato público por parte de la ciudadanía. En este contexto, la moral puede ser un factor de enorme trascendencia para que el estudiante adopte nuevos comportamientos, de tal modo que pueda asumir posiciones altruistas, de compromiso y de 
responsabilidad social; además de contar con el apoyo de programas experienciales de corto plazos (Wynveen, et al., 2012).

Sin embargo, tampoco debe dejarse de lado, la percepción acerca de la falta de compromiso de parte de las instituciones y autoridades responsables en el ámbito de su competencia, más allá de la conducta de las personas y las malas prácticas ambientales, como el mal manejo de los residuos sólidos o el descuido de espacios con vegetación como parques y jardines (AhumadaTello et al., 2018). Este aspecto cobra especial relevancia porque adjudicarles a otros la responsabilidad individual, influye de manera negativa en el compromiso que como individuos se tiene para con el cuidado del medio ambiente en la vida diaria.

La conducta y la actitud ambiental responsable son entendidas dentro del contexto sociocultural en que se producen; esto hace posible identificar las oportunidades que ofrecen los programas sociales, experiencias interuniversitarias, la articulación de aspectos ambientales con los educativos; el cuestionamiento del mal uso de los recursos y las prácticas que dan origen a los problemas ambientales; así como la búsqueda de respuestas comprensivas y holísticas. Aunado a ello, los resultados obtenidos proporcionan elementos orientadores para el análisis de las situaciones de conductas y actitudes ambientales responsables, de los tipos de conocimientos presentes, de los marcos de referencia y del modo de razonar de los estudiantes universitarios. En estas circunstancias, se puede inferir que tanto la conducta como la actitud ambiental responsable mantienen fuertes vínculos, que, si bien han sido estudiados, no obstante, son pocas las investigaciones en la población universitaria; por ende, los datos mostrados generan un nuevo aporte a la comunidad científica.

$\mathrm{Si}$ bien existieron ciertas limitaciones que deben ser tomadas en cuenta, para investigaciones posteriores, hubiese sido preferible que el porcentaje de evaluados de acuerdo con el sexo, sea en lo posible cercano; de modo que se plantease un resultado comparativo significativo por la equidad muestral. Además, aunque no existen instrumentos que midan la conducta y actitud ambiental responsable en el contexto peruano, los autores procedieron a elaborar el mismo para llevar a cabo la investigación, por lo que en el futuro este aspecto puede constituirse en una fortaleza por la confiabilidad y el uso frecuente que pueda darse a dicho instrumento. De manera que, este estudio marca un precedente para estudios de estos fenómenos. 


\section{Conclusión}

El estudiante universitario es un actor fundamental en la estructura de la sociedad, de tal manera que la educación que forma un pensamiento, creencia, cultura e involucra las emociones y motivaciones para con el cuidado ambiental, puede generar actitudes responsables a favor de un desarrollo social más equitativo. Se ha demostrado que existe una importante relación entre las actitudes responsable y la conducta proambiental; de modo que, su vínculo se genera por esquemas, conceptos y realidades los cuales promueven la intención conductual. Sin embargo, la intención como un fenómeno previo a la conducta debe ser estimulado por los factores involucrados en la actitud. Estos factores se desenvuelven en un complejo procesamiento cognitivo que generan voluntad de ayuda y responsabilidad con el medio ambiente. Es así como los elementos de la actitud responsable impulsan en gran medida la conducta proambiental. Por otro lado, los factores de la conducta proambiental muestran una relación significativa, lo que permite concluir que las conductas pueden verse influenciadas por la realidad y el pensamiento del estudiante a la hora de enfrentarse a la complejidad de un problema ambiental.

Sobre esta base, se sugiere que estudios posteriores, desarrollen investigaciones explicativas para comprender el fenómeno conductual y psicológico del estudiante frente a problemas sociales como la contaminación ambiental. Además, es necesario el estudio de modelos predictivos y experimentales que brinden estrategias aplicativas para generar actitud y conducta ambiental responsable en beneficio de la sociedad. Por último, se sugiere que las instituciones educativas consideren como primordial el estudio de los fenómenos ambientales desde las diversas áreas del conocimiento; y promover los aportes multidisciplinarios.

\section{Referencias}

Ahumada-Tello, E., Ravina-Ripoll, R. \& López-Regalado, M. (2018). Responsabilidad Social Universitaria. Desarrollo de competitividad organizacional desde el proceso educativo. Actualidades Investigativas en Educación, 18(3), 359-

389. https://dx.doi.org/10.15517/aie.v18i3.34213

Albalá, M. Á. \& Maldonado, A. (2018). Factores psicosociales implicados en la actitud hacia la participación del futuro profesorado en Madrid. Investigaciones en Psicología, 23(2): 5462. https://doi.org/10.32824/investigpsicol.a23n2a5 
Álvarez, P. \& Vega, P. (2009). Actitudes ambientales y conductas sostenibles. Implicaciones para la educación ambiental. Revista de Psicodidáctica, 14(2):245-260. Disponible desde internet

en https://ruc.udc.es/dspace/bitstream/handle/2183/19179/Vega_Marcote_2009_Actitudes_ ambientales_\%20conductas_sostenibles.pdf?sequence=3\&isAllowed=y

Álvarez-Lires, M.M.; Arias-Correa, A.; Lorenzo-Rial, M.A.; \& Serrallé-Marzoa, F. (2017). Educación para la Sustentabilidad: Cambio Global y Acidificación Oceánica. Formación universitaria. 10(2):89-102. https://dx.doi.org/10.4067/S0718-50062017000200010

Amérigo, M.; García, J. A.; \& Sánchez, T. (2012). Actitudes y comportamiento hacia el medio ambiente natural. Salud medioambiental y bienestar emocional. Universitas Psuchologien. 12(3):845-856. https://doi.org/10.11144/Javeriana.UPSY12-3.acma

Amérigo, M.; García, J.; \& Côrtes, P. (2017). Analysis of environmental attitudes and behaviors: an exploratory study with a sample of brazilian university students. Ambiente \& Sociedade. 20(3):1-20. https://doi.org/10.1590/1809-4422asoc300r1v2032017

Balderjahn, I. (1988). Personality variables and environmental attitudes as predictors of ecologically responsible consumption patterns. Journal of Business Research, 17(1), 5156. https://doi.org/10.1016/0148-2963(88)90022-7

Barreto, I.; \& Neme, S. (2014). Eficacia de tácticas de influencia en la intención de conducta proambiental. Revista Latinoamericana de Psicología. 46(2):111-116. https://doi.org/10.1016/S0120-0534(14)70014-7

Bermúdez, A.; Terán, M.; Caldera, R.V.; \& Castillo, M. (2011). Estrategias instruccionales para promover actitudes proambientales en estudiantes universitarios. Investigación Arbitrados, 15(51), 409-421. Disponible desde internet en https://dialnet.unirioja.es/servlet/articulo?codigo=6357669

Calixto, R. (2012). Investigación en Educación Ambiental. Revista Mexicana de Investigación Educativa, 17(55), 1019-1033. Disponible desde internet en http://www.scielo.org.mx/pdf/rmie/v17n55/v17n55a2.pdf

Campos, M. L.; Oasquali, C.; \& Peinado, S. (2015). Evaluación psicométrica de un instrumento de medición de actitudes proambientales en escolares venezolanos. Revista Paradigma, 29(2), 135-156. Disponible desde internet en 
https://www.researchgate.net/publication/262590087_Evaluacion_psicometrica_de_un_i nstrumento_de_medicion_de_actitudes_pro_ambientales_en_escolares_venezolanos

Chakraborty, A.; Pratap, M.; \& Roy, M. (2017). A study of goal frames shaping pro-environmental behaviour in university students. International Journal of Sustainability in higher Education. https://doi.org/10.1108/IJSHE-10-2016-0185

Choon, S., Ong, H. \& Tan, S. (2019). Does risk perception limit the climate change mitigation behaviors? Environ Dev Sustain 21, 1891-1917. https://doi.org/10.1007/s10668-0180108-0

Cortés-Peña, O. F. (2016). Comportamiento proambiental y desarrollo económico sustentable en jóvenes universitarios. Opción, 32(9), 387-407. Recuperado de la base de datos Dialnet. Disponible desde internet en https://dialnet.unirioja.es/servlet/articulo?codigo $=5891174$

Dourojeanni, M. J. (2019). Amazonia Peruana ¿Qué futuro? Ed. Pronaturaleza y Universidad Nacional Enrique Guzmán y Valle. Grijley Lima. 395. Disponible desde internet en https://www.academia.edu/39962447/Amazonia_Qu\%C3\%A9_Futuro

Espino-Román, P.; Olanguez-Torres, E.; \& Davizon-Castillo, Y. A. (2015). Análisis de la Percepción del Medio Ambiente de los Estudiantes de Ingeniería en Mecatrónica. Formación universitaria, 8(4). http://dx.doi.org/10.4067/S0718-50062015000400006

Fuentealba, M.; \& Soto, L. (2016). Valoración actitudinal frente a temas ambientales. Revista Luna Azul, 43, 448-467. Disponible desde internet en http://www.scielo.org.co/pdf/luaz/n43/n43a19.pdf

Gomera, A. (2008). La conciencia ambiental como herramienta para la educación ambiental: Conclusiones y reflexiones de un estudio en el ámbito universitario. Centro Nacional de Educación ambiental. p. 6. Disponible desde internet en https://www.miteco.gob.es/es/ceneam/articulos-de-opinion/2008_11gomera1_tcm30163624.pdf

Hernández, R. \& Mendoza, C. (2018). Metodología de la Investigación: las rutas cuantitativa, cualitativa y mixta. Ciudad de México: McGraw-Hill/Interamericana Editores.

Herrera-Mendoza, K.; Acuña, M.; Ramírez, M. J.; \& De la hoz, M. (2016). Actitud y conducta proecológica de jóvenes universitarios. Opción, 32(13), 456-477. Disponible desde internet en https://dialnet.unirioja.es/servlet/articulo?codigo $=5844677$ 
Janmaimool, P.; \& Khajohnmanee, S. (2018). Enhancing university students' global citizenship, public mindedness, and moral quotient for promoting sense of environmental responsibility and pro-environmental behaviours. Environment, Development and Sustainability. https://doi.org/10.1007/s10668-018-0228-6

Leiva, F. (2020). Educación Ambiental para el poblador del distrito de Casa Grande en el manejo de residuos sólidos urbanos entre julio a diciembre del año 2019. Arnaldoa, 27 (1):323334. http://doi.org/10.22497/arnaldoa.271.27120

Lucie, S. (2010). Educación científica y educación ambiental: un cruce profundo. Enseñanza de las Ciencias, 28(1): 5-18. Disponible desde internet en https://www.raco.cat/index.php/Ensenanza/article/view/189092/353371

Medina, R., Franco, M., Torres, L., Velázquez, K., Valencia, M. \& Valencia, A. (2017). University social responsibility in the current knowledge society. MediSur, 15(6), 786791. http://scielo.sld.cu/scielo.php?script=sci_arttext\&pid=S1727897X2017000600006\&lng=es\&tlng=en.

Olaguez-Torres, E.; Espino-Román, P.; Acosta-Pérez, K.; \& Méndez-Barceló, A. (2019). Plan de acción a partir de la percepción en estudiantes de la Universidad Politécnica de Sinaloa ante el reciclaje de residuos sólidos y la educación ambiental. Formación universitaria, 12(3). http://dx.doi.org/10.4067/S0718-50062019000300003

Olivera, E. S. (2020). Actitudes hacia la investigación de bachilleres en administración y psicología de una universidad peruana. Revista de Ciencias Sociales y Humanidades, Chakiñan. https://doi.org/10.37135/chk.002.11.05

Onokala, U.; Banwo, A.; \& Okeowo, F. (2018). Predictors of Pro-Environmental Behavior; A Comparison of University Students in the United States and China. Journal of Management and Sustainability, 8(1). http://doi.org/10.5539/jms.v8n1p127

Palacios, J. R., Bustos, M.; \& Soler, L. (2015). Factores socioculturales vinculados al comportamiento proambiental en jóvenes. Revista de Psicología, 24(1), 1-16. Doi: http://dx.doi.org/10.5354/0719-0581.2015.36900

Pulido, V. (2017), Ecología, Lima, Perú, Fondo Editorial de la Universidad Inca Garcilaso de la Vega. $280 \mathrm{pp}$. 
Pulido, V; \& Olivera, E. (2018). Aportes pedagógicos a la educación ambiental: una perspectiva teórica. Revista de investigación Altoandina. 20(3):

333346. http://dx.doi.org/10.18271/ria.2018.397

Rivera-jacinto, M.; \& Rodríguez-Ulloa, C. (2009). Actitudes y comportamientos ambientales en estudiantes de enfermería de una universidad pública del norte del Perú. Revista Peruana de Medicina Experimental y Salud Pública, 26(3), 338-342. Disponible desde internet en http://www.scielo.org.pe/pdf/rins/v26n3/a12v26n3.pdf

Schultz, W. (2000). Empathizing with nature: The effects of perspective taking on concern for environmental issues. Journal of Social Issues, 56, 391-406. Disponible desde internet en https://citeseerx.ist.psu.edu/viewdoc/download?doi=10.1.1.477.4138\&rep=rep1\&type=p df

Schultz, W. (2001). The structure of environmental concern: Concern for self, other people, and the biosphere. Journal of Environmental Psychology, 21(4): 327-339. https://doi.org/10.1006/jevp.2001.0227

Sevillano, V; \& Olivos, P. (2019). Comportamiento social y ambiente: Influencias de las normas sociales en la conducta ambiental. Psychologist Papers, 40(3), 182-189. https://doi.org/10.23923/pap.psicol2019.2898

Torres, H. F., Suárez, L., \& Dueñas, J. O. (2016). El diálogo y lo visual como mediadores de relaciones entre el entorno y la apreciación-creación. Revista Conrado, 12(56): 47-54. Disponible desde internet en https://conrado.ucf.edu.cu/index.php/conrado/article/view/402

Torres-Maya, H. F.; Suárez, A. A.; Suárez-Vivas, L.; \& Verdecia-Marín, M. (2019). La apreciación visual del entorno natural en la Educación Ambiental. Revista Científica Agroecosistemas, 7(2), 172-181. https://aes.ucf.edu.cu/index.php/aes/article/view/291/314

Umai-unión Mexicana de Asociación de Ingenieros. (2009). Foro Panamericano sobre Contribuciones de la Ingeniería al Mejoramiento del Medio Ambiente. Disponible desde internet en http://www.umai.org.mx/Noticias/2009/ForoAPI/docspa.pdf

UNESCO. (1975). La Carta de Belgrado: un marco general para la educación ambiental. Disponible desde internet en http://unesdoc.unesco.org/images/0001/000177/017772SB.pdf 
UNESCO. (1978). Conferencia Intergubernamental sobre Educación Ambiental. Tbilisi (URSS). Paris, Francia: UNESCO. Disponible desde internet en https://agua.org.mx/wpcontent/uploads/2018/01/Conferencia-Intergubernamental-sobre-Educaci\%C3\%B3nAmbiental-Tbilisi-URSS.pdf

Vega-Marcote, P. (2008). Actitudes ambientales y conductas sostenibles. Implicaciones para la Educación Ambiental. Rev. Psicodidáctica. 14(2):245-260. Disponible desde internet en https://ruc.udc.es/dspace/bitstream/handle/2183/19179/Vega_Marcote_2009_Actitudes_ ambientales_\%20conductas_sostenibles.pdf?sequence=3\&isAllowed=y

Vicente-Molina, M.; Fernández-Sáinz, A.; Izagirre-Olaizola, J. (2013). Environmental knowledge and other variables affecting pro-environmental behaviour; comparison of university students from emerging and advanced countries. Journal of Cleaner Production, 61, 130138. https://doi.org/10.1016/j.jclepro.2013.05.015

Vila-Tojo, S., Andrade, E., Sabucedo, J. M., González-García, S., Moreira, M. T., \& Feijoo, G. (2019). Revisión sobre las características metodológicas y la eficacia de intervenciones orientadas a reducir el consumo de agua. Universitas Psychologica, 18(5), 1-15. https://doi.org/10.11144/Javeriana.upsy18-5.rscm

Villadiego-Lordy, J.; Huffman-Schwocho, D.; Guerrero, S.; Cortecero-Bossio, A. (2017). Base pedagógica para generar un modelo no formal de educación ambiental. Revista Luna Azul, 44, 316-333. http://dx.doi.org/10.17151/luaz.2017.44.19

WORLD BANK, IFC \& MIGA. (2016). World Bank Group Climate Change Action Plan 20162020. World Bank, Washington DC. License: Creative Commons Attribution CC BY 3.0 IGO. 74 pp. Disponible desde internet en https://openknowledge.worldbank.org/bitstream/handle/10986/24451/K8860.pdf

Wynveen, C.; Kyle, G.; Tarrant, M. (2012). Study abroad experiences and global citizenship: $\begin{array}{lllll}\text { fostering } & \text { proenvironmental 334-352. }\end{array}$ https://doi.org/10.1177/1028315311426782

Yilmaz, V., Can, Y. (2020). Impact of knowledge, concern and awareness about global warming and global climatic change on environmental behavior. Environ Dev Sustain 22, 62456260. https://doi.org/10.1007/s10668-019-00475-5

Yupanqui, D. (2017). Actitud hacia el consumo de alcohol en estudiantes de psicología de una universidad privada de Lima Metropolitana. Revista Peruana de Psicología y Trabajo 
Social,

$6(2)$.

Disponible

desde

internet

en

http://revistas.uigv.edu.pe/index.php/psicologia/article/view/507

Anexo 1

Cuestionario Actitud ambiental responsable Conductas proambientales

\begin{tabular}{|c|c|c|c|c|c|c|}
\hline & Actitud hacia el ambiente & 1 & 2 & 3 & 4 & 5 \\
\hline 1 & $\begin{array}{l}\text { El dióxido de carbono emitido por los autos contamina el medio } \\
\text { ambiente }\end{array}$ & & & & & \\
\hline 2 & El humo de los buses es perjudicial para el aire que respiramos & & & & & \\
\hline 3 & Es nuestro deber cuidar el aire & & & & & \\
\hline 4 & Las fábricas son fuentes de contaminación al aire & & & & & \\
\hline 5 & El aire contaminado daña la salud de las personas & & & & & \\
\hline 6 & Se deben cuidar la vegetación por ser parte esencial de la vida & & & & & \\
\hline 7 & El aire puro es necesario para poder vivir & & & & & \\
\hline 8 & Faltan áreas verdes por esta zona & & & & & \\
\hline 9 & $\begin{array}{l}\text { Las empresas con grandes fábricas son causa de la contaminación } \\
\text { ambiental }\end{array}$ & & & & & \\
\hline 10 & Las personas no les interesa el cuidado ambiental & & & & & \\
\hline 11 & La vegetación es importante porque brinda oxígeno a la tierra & & & & & \\
\hline 12 & Regar las áreas verdes produce mucho gasto de consumo en el agua & & & & & \\
\hline 13 & $\begin{array}{l}\text { La contaminación se ve reducida por la presencia de árboles y } \\
\text { plantas }\end{array}$ & & & & & \\
\hline 14 & En mi zona, las construcciones han reducido la vegetación & & & & & \\
\hline 15 & $\begin{array}{l}\text { Cuidar el medio ambiente reduciría las probabilidades de reducir el } \\
\text { calentamiento global }\end{array}$ & & & & & \\
\hline 16 & $\begin{array}{l}\text { Las personas no toman conciencia de su responsabilidad como } \\
\text { cuidadores de la naturaleza }\end{array}$ & & & & & \\
\hline 17 & La basura puede generar contaminación ambiental & & & & & \\
\hline 18 & Las autoridades deben cuidar las áreas verdes & & & & & \\
\hline
\end{tabular}

$1=$ Totalmente en desacuerdo

$2=$ En desacuerdo

$3=\mathrm{Ni}$ de acuerdo ni en desacuerdo

$4=$ De acuerdo

$5=$ Totalmente de acuerdo 


\section{Anexo 2}

\section{Cuestionario Conducta proambientales}

\begin{tabular}{|c|c|c|c|c|c|c|}
\hline & Intención de conductas proambientales & 1 & 2 & 3 & 4 & 5 \\
\hline 1 & $\begin{array}{l}\text { Se debe enseñar a las personas a botar la basura en bolsas } \\
\text { diferenciadas para plástico, papeles y orgánicos }\end{array}$ & & & & & \\
\hline 2 & Puedo tomar acciones para cuidar las áreas verdes & & & & & \\
\hline 3 & Al limpiar la casa pongo la basura en bolsas diferenciables & & & & & \\
\hline 4 & Deseo sembrar plantas y árboles & & & & & \\
\hline 5 & No boto basura en la calle & & & & & \\
\hline 6 & Pongo las bolsas de basura en lugares fijos & & & & & \\
\hline 7 & $\begin{array}{l}\text { Las personas deben comunicarse con las autoridades para el cuidado } \\
\text { ambiental }\end{array}$ & & & & & \\
\hline 8 & Reciclo botellas de plástico, papeles y otros objetos & & & & & \\
\hline 9 & No utilizó productos que puedan contaminar el medio ambiente & & & & & \\
\hline 10 & Tengo plantas en casa & & & & & \\
\hline 11 & Cuido las áreas verdes cerca de mi hogar & & & & & \\
\hline 12 & Las personas pueden crear grupos para cuidar el medio ambiente & & & & & \\
\hline 13 & No utilizó productos químicos en casa & & & & & \\
\hline
\end{tabular}

$1=$ Totalmente en desacuerdo

$2=$ En desacuerdo

$3=$ Ni de acuerdo ni en desacuerdo

$4=$ De acuerdo

$5=$ Totalmente de acuerdo 